\title{
Uma abordagem mesoescala para simulação estrutural de tubos flexíveis
}

\author{
Carvalho, R. R. ${ }^{1^{*}}$; Loeffler, C. ${ }^{2}$; \\ Programa de Pós-Graduação em Engenharia Mecânica, Universidade Federal do Espírito Santo, Vitória, ES, Brasil. \\ Departamento de Engenharia Mecânica, Universidade Federal do Espírito Santo, Vitória, ES, Brasil. \\ * e-mail: rodrigo.r.carvalho@aluno.ufes.br
}

\begin{abstract}
Resumo
Neste trabalho é mostrada uma abordagem analítica para o problema de um sistema formado por tubos compostos por paredes espessas de diferentes propriedades visando a construção de uma matriz de rigidez em meso escala. Analisa-se uma solicitação imposta ao sistema na forma de pressão interna e externa. A partir de manipulações da Solução de Lamé, associada com as condições de contorno do problema, será obtida uma matriz de flexibilidade equivalente para um sistema composto de um par de camadas. Em seguida, será realizada a extrapolação dessa matriz para um sistema composto por $\mathrm{n}$ pares de camadas.
\end{abstract}

\begin{abstract}
In this paper, it will be presented an analytical approach for the problem of a multilayered tube aiming a construction of a stiffness matrix concerning a mesoscale level. It will be analyzed a system subjected to an internal and external pressure load. From the Lamé Solution, associated with the boundary conditions of the problem, it will be achieved a flexibility matrix for a system composed by one pair of layers. Then, it will be shown the extrapolation of this Matrix for a system formed by $n$ pair of layers.
\end{abstract}

Keywords: Tubos multicamadas, Matriz de Flexibilidade, Solução de Lamé.

\section{Introdução}

Com o crescente desenvolvimento tecnológico, aumentou também o uso de simulações numéricas. Com problemas a cada dia mais complexos, a diminuição do custo computacional e a necessidade de altas precisões nos métodos de solução tornam-se mais frequentes. Por outro lado, há exigência de um maior rigor da descrição do meio, que em alguns casos não mais pode ser considerado como idealmente contínuo. Porém, a introdução de detalhes ou particularidades em escala reduzida para todo o domínio pode tornar inviável a sua simulação computacional. O método multiescala tem por objetivo reduzir esses custos computacionais e/ou alcançar valores mais próximos dos reais. Além disso, o método contribui em melhorar a ponte de conexão entre o campo da mecânica dos materiais e o campo da ciência dos materiais.

Há algumas variantes no procedimento, que pode ser feito em diferentes padrões de expansão [1]. Contudo, grosso modo, o método multiescala consiste no levantamento do comportamento estrutural, via propriedades constitutivas e variáveis de estado, num nível mais fundamental - que pode ser nano, micro ou meso - para posterior operacionalização num nível macro. No entanto, para se proceder ao entrelaçamento das diferentes característicos do objeto 
de estudo num mesmo patamar, é preciso estabelecer modelos que se compatibilizem adequadamente nesta transição, além de ferramentas de cálculo que operem em diferentes escalas.

Diversos são os campos de aplicação da análise multiescala. No caso de tubos multicamadas, exemplifica-se seu uso na análise de risers de petróleo como em [4], nanofios em semicondutores em [5] ou compósitos com revestimentos em fibra, como em [6]. Em [7], utiliza-se microescala para a obtenção das propriedades de cada camada constituída de compósitos, utilizando informações como as fibras e a matriz desses compósitos, e realiza-se então a simulação em macroescala com os dados obtidos da microescala.

Em consonância com essa idéia buscou-se aqui desenvolver uma formulação analítica em termos de uma matriz de influência para o problema formado por tubos compostos de paredes espessas, também denominados de tubos multicamadas. Essa matriz de influência local, que pode ser enquadrada num nível de meso escala, torna-se necessária para o desenvolvimento de um método numérico que resolva o problema na escala global com menor custo. Existem abordagens aparentemente semelhantes, como em [8], [9] e [2]. No entanto, apesar de apresentarem a solução de vários exemplos envolvendo tubos multicamadas, essas referências não explicitaram de forma clara como calcularam e qual era a composição da matriz de resistência ou flexibilidade utilizada.

\section{Metodologia}

Como se trata de um problema de análise de tensões, se utilizou como ponto de partida a solução de tubos de paredes espessas proposta por Gabriel Lamé (1795 1870), conhecidas como as Soluções de Lamé. Esse é o ponto de partida mais generalizado e também o mais utilizado por diversas publicações envolvendo tubos de paredes espessas submetidos aos mais diversos tipos de carregamentos [2].

De acordo com [3], a formulação da solução de Lamé é dada por:

$$
\begin{gathered}
\sigma_{R}=\frac{P_{i} r_{i}{ }^{2}-P_{e} r_{e}{ }^{2}}{r_{e}{ }^{2}-r_{i}{ }^{2}}-\frac{r_{i}{ }^{2} r_{e}{ }^{2}\left(P_{i}-P_{e}\right)}{r_{e}{ }^{2}-r_{i}{ }^{2}} \cdot \frac{1}{r^{2}} \\
\sigma_{\theta}=\frac{P_{i} r_{i}{ }^{2}-P_{e} r_{e}{ }^{2}}{r_{e}{ }^{2}-r_{i}{ }^{2}}+\frac{r_{i}{ }^{2} r_{e}{ }^{2}\left(P_{i}-P_{e}\right)}{r_{e}{ }^{2}-r_{i}{ }^{2}} \cdot \frac{1}{r^{2}} \\
\delta_{R}=\frac{1-v}{E} \cdot \frac{\left(P_{i} r_{i}{ }^{2}-P_{e} r_{e}{ }^{2}\right)}{r_{e}{ }^{2}-r_{i}{ }^{2}} \cdot r+\frac{1+v}{E} \cdot \frac{r_{i}{ }^{2} r_{e}{ }^{2}\left(P_{i}-P_{e}\right.}{r_{e}{ }^{2} r_{i}{ }^{2}}
\end{gathered}
$$

Nas expressões anteriores, $\sigma_{R}$ e $\sigma_{\theta}$ são respectivamente as tensões radiais e circunferenciais; $\delta_{R}$ é o deslocamento radial; $P_{1}$ e $P_{e}$ são respectivamente as pressões interna e externa, enquanto $r_{i}$ e $r_{e}$ são os raios interno e externo do tubo. As propriedades físicas $E$ e $v$ são o módulo de elasticidade longitudinal e o coeficiente de Poisson, enquanto $r$ é o raio qualquer num ponto da tubulação.

A solução de Lamé é obtida por meio da aplicação das equações constitutivas da resistência dos materiais aplicadas em coordenadas cilíndricas, levando como consideração o deslocamento circunferencial nulo e simetria axial das tensões e deslocamentos. Ela é amplamente utilizada no estudo de problemas envolvendo tubos de paredes espessas.

Será analisado inicialmente o sistema constituído por um tubo formado de duas camadas totalmente vinculadas entre si, sem que haja qualquer forma de descolamento ou escorregamento entre as camadas.

Dessa forma, incorre-se que haja uma condição de compatibilidade de deslocamentos na interface das camadas, que será aplicado como uma das condições de contorno na análise. Além disso, será definido que o sistema está submetido apenas a pressão estática interna e externa, sem aplicação de esforços torcionais e axiais.

O desenvolvimento matemático foi realizado na direção radial do sistema.

Em um segundo momento, será realizada a expansão da análise para um sistema formado por um tubo com 4 camadas, e será demostrado que é possível expandir para um sistema formado por $2^{*} n$ camadas de forma 
metódica e consistente, fazendo com que seja possível a implementação de um método numérico para a resolução das equações.

Inicialmente, analisa-se um conjunto formado por dois tubos. Para o deslocamento na camada interna (camada 1) $\delta_{1}$, tem-se:

$\delta_{1}=\left[\frac{\left(1-v_{1}\right) r_{i}^{2} r+\left(1+v_{1}\right) \frac{r_{i}^{2} r_{c}^{2}}{r}}{E_{1}\left(r_{c}^{2}-r_{i}^{2}\right)}\right] P_{i^{-}}\left[\frac{\left(1-v_{1}\right) r_{c}^{2} r+\left(1+v_{1}\right) \frac{r_{i}^{2} r_{c}^{2}}{r}}{E_{1}\left(r_{c}{ }^{2}-r_{i}^{2}\right)}\right] P_{c}$

Repetindo-se para a camada externa (camada 2) $\delta_{2}$ :

$\delta_{2}=\left[\frac{\left(1-v_{2}\right) r_{c}{ }^{2} r+\left(1+v_{2}\right) \frac{r_{c}{ }^{2} r_{o}{ }^{2}}{r}}{E_{2}\left(r_{o}{ }^{2}-r_{c}{ }^{2}\right)}\right] P_{c}-\left[\frac{\left(1-v_{2}\right) r_{o}{ }^{2} \boldsymbol{r}+\left(1+v_{2}\right) \frac{r_{c}{ }^{2} r_{o}{ }^{2}}{r}}{E_{2}\left(r_{o}{ }^{2}-r_{c}{ }^{2}\right)}\right] P_{o}$

Com isso, observa-se que a pressão de contato na interface $P_{c}$ é desconhecida. Para sua obtenção, será utilizada a condição de compatibilidade acima descrita, determinada por meio de $\left.\delta_{1}\right|_{r_{c}}=\left.\delta_{2}\right|_{r_{\varepsilon}}$. Com isso, é possível demostrar por meio de manipulação matemática que:

$$
P_{c}=A P_{i}+B P_{o}
$$

Sendo $A$ e $B$ constantes determinadas por meio das propriedades elásticas e geométricas do sistema:

$$
\begin{aligned}
& A=\frac{\frac{2 r_{i}{ }^{2} r_{c}}{E_{1}\left(r_{c}{ }^{2}-r_{i}{ }^{2}\right)}}{\frac{\left(1-v_{1}\right) r_{c}{ }^{3}+\left(1+v_{1}\right) r_{i}{ }^{2} r_{c}}{E_{1}\left(r_{c}{ }^{2}-r_{i}{ }^{2}\right)}+\frac{\left(1-v_{2}\right) r_{c}{ }^{3}+\left(1+v_{2}\right) r_{o}{ }^{2} r_{c}}{E_{2}\left(r_{o}{ }^{2}-r_{c}{ }^{2}\right)}} \\
& B=\frac{\frac{2 r_{o}{ }^{2} r_{c}}{E_{2}\left(r_{o}{ }^{2}-r_{c}{ }^{2}\right)}}{\frac{\left(1-v_{1}\right) r_{c}{ }^{3}+\left(1+v_{1}\right) r_{i}^{2} r_{c}}{E_{1}\left(r_{c}{ }^{2}-r_{i}{ }^{2}\right)}+\frac{\left(1-v_{2}\right) r_{c}{ }^{3}+\left(1+v_{2}\right) r_{o}{ }^{2} r_{c}}{E_{2}\left(r_{o}{ }^{2}-r_{c}{ }^{2}\right)}}
\end{aligned}
$$

Substituindo a equação 6 nos deslocamentos de cada tubo e avaliando-os na parede interna do sistema e na parede externa do sistema, mostra-se que:

$$
u_{i}=\psi_{11} P_{i}+\psi_{12} P_{o}
$$

\section{Blucher}

$$
u_{o}=\psi_{21} P_{i}+\psi_{22} P_{o}
$$

Sendo $\psi_{11}, \psi_{12}, \psi_{21}$ e $\psi_{22}$ constantes dependentes das propriedades elásticas e geométricas do sistema:

$$
\begin{gathered}
\psi_{11}=\frac{\left(1-v_{1}\right) r_{i}^{3}+\left(1+v_{1}\right) r_{i} r_{c}{ }^{2}-2 r_{c}{ }^{2} r_{i} A}{E_{1}\left(r_{c}{ }^{2}-r_{i}^{2}\right)} \\
\psi_{12}=-\left[\frac{2 r_{c}^{2} r_{i} B}{E_{1}\left(r_{c}^{2}-r_{i}^{2}\right)}\right] \\
\psi_{21}=\frac{2 r_{c}^{2} r_{o} A}{E_{2}\left(r_{o}{ }^{2}-r_{c}{ }^{2}\right)}
\end{gathered}
$$

$$
\psi_{22}=\frac{2 r_{c}{ }^{2} r_{o} B-\left(1-v_{2}\right) r_{o}{ }^{3}-\left(1+v_{2}\right) r_{c}{ }^{2} r_{o}}{E_{2}\left(r_{o}{ }^{2} r_{c}{ }^{2}\right)}
$$

Dessa forma, pelo sistema de equações 9 e 10, é possível montar a Matriz de Flexibilidade de um sistema composto por dois tubos distintos:

$$
\left\{\begin{array}{l}
u_{i} \\
u_{o}
\end{array}\right\}=\left[\begin{array}{ll}
\psi_{11} & \psi_{12} \\
\psi_{21} & \psi_{22}
\end{array}\right]\left\{\begin{array}{l}
P_{i} \\
P_{o}
\end{array}\right\}
$$

Essa matriz poderá ser utilizada como parte da implementação numérica do problema, por meio do método dos elementos de contorno, em uma pesquisa futura.

Em seguida, buscou-se a análise para sistemas formados por mais de 1 par de tubos $(2,4,6 \ldots$ tubos), de forma a se obter uma matriz para um problema mais generalizado e de forma metódica, passível de implementação.

O modelo foi proposto pelo acoplamento aos pares conforme mostra a Figura 1.

Para o par de tubos 1 e 2, a definição da Matriz de Flexibilidade segue conforme anteriormente proposto:

$$
\left\{\begin{array}{l}
u_{1} \\
u_{3}
\end{array}\right\}=\left[\begin{array}{ll}
\psi_{11} & \psi_{12} \\
\psi_{21} & \psi_{22}
\end{array}\right]\left\{\begin{array}{l}
P_{1} \\
P_{3}
\end{array}\right\}
$$



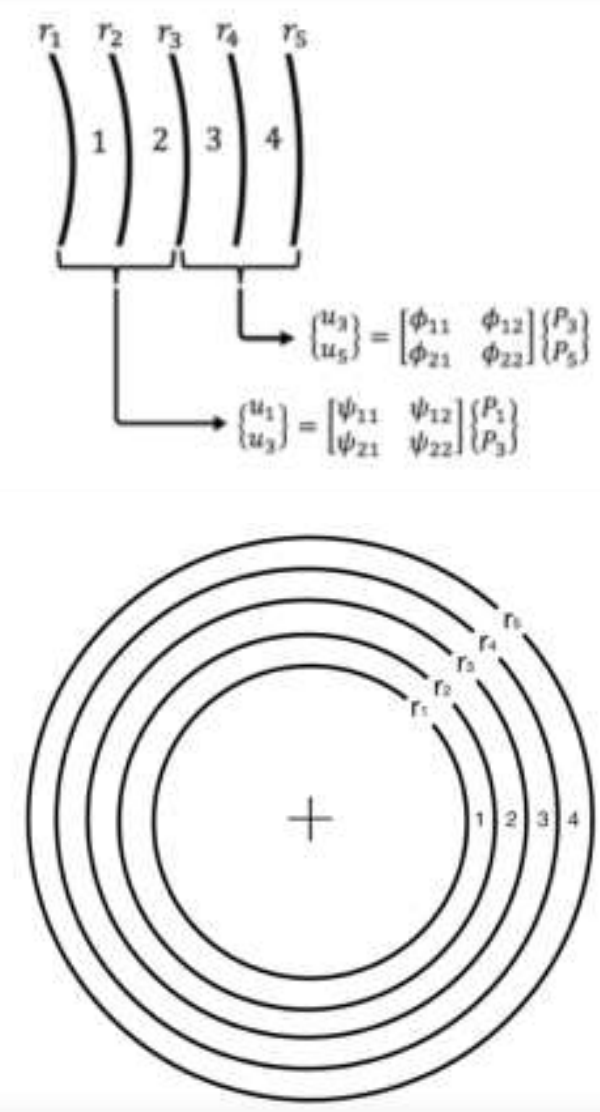

Figura 1 - Representação esquemática do acoplamento da tubulação.

Também para o par de tubos 3 e 4, tem-se:

$$
\left\{\begin{array}{l}
u_{3} \\
u_{5}
\end{array}\right\}=\left[\begin{array}{ll}
\phi_{11} & \phi_{12} \\
\phi_{21} & \phi_{22}
\end{array}\right]\left\{\begin{array}{l}
P_{3} \\
P_{5}
\end{array}\right\}
$$

Com as duas matrizes de flexibilidade definidas, as equações disponíveis no problema vêm a seguir:

$$
\begin{aligned}
& u_{1}=\psi_{11} P_{1}+\psi_{12} P_{3} \\
& u_{3}=\psi_{21} P_{1}+\psi_{22} P_{3} \\
& u_{3}=\phi_{11} P_{3}+\phi_{12} P_{5} \\
& u_{5}=\phi_{21} P_{3}+\phi_{22} P_{5}
\end{aligned}
$$

Por meio da condição de compatibilidade de deslocamentos aplicada ao deslocamento $u_{3}$, além da aplicação da equação 6 desenvolvida para a pressão de contato aplicada a $P_{a}$, é possível demonstrar por meio de manipulações matemáticas o seguinte sistema de equações:

$$
\begin{gathered}
\boldsymbol{u}_{\mathbf{1}}=\boldsymbol{\varphi}_{\mathbf{1 1}} \boldsymbol{P}_{\mathbf{1}}+\boldsymbol{\varphi}_{\mathbf{1 2}} \boldsymbol{P}_{\mathbf{5}} \\
u_{5}=\varphi_{21} P_{1}+\varphi_{22} P_{5}
\end{gathered}
$$

Sendo $\varphi_{11}, \varphi_{12}, \varphi_{21}$ e $\varphi_{22}$ constantes dependentes somente de propriedades elásticas e geométricas.

Com base nas equações 22 e 23, é possível montar nova matriz de flexibilidade para o sistema formado por 4 camadas. Com essa matriz, é possível analisar o comportamento do tubo multicamada nas faces internas e externas sem depender do comportamento nas camadas internas:

$$
\left\{\begin{array}{l}
u_{1} \\
u_{5}
\end{array}\right\}=\left[\begin{array}{ll}
\varphi_{11} & \varphi_{12} \\
\varphi_{21} & \varphi_{22}
\end{array}\right]\left\{\begin{array}{l}
P_{1} \\
P_{5}
\end{array}\right\}
$$

Além disso, outro ponto importante é realizado com o somatório das equações 19 e 20. Com essa manipulação tem-se a seguinte matriz de flexibilidade:

$$
\left\{\begin{array}{l}
u_{1} \\
u_{2} \\
u_{2}
\end{array}\right\}=\left[\begin{array}{ccc}
\psi_{12} & \psi_{12} & 0 \\
\frac{\psi_{21}}{2} & \frac{\left(\psi_{12}+\phi_{22}\right)}{2} & \frac{\phi_{12}}{2} \\
0 & \phi_{12} & \phi_{12}
\end{array}\right]\left\{\begin{array}{c}
P_{2} \\
P_{x} \\
P_{2}
\end{array}\right\}
$$

Conhecidas os parâmetros por meio da matriz 24, é possível obter os valores da pressão de contato e do deslocamento na interface por meio da matriz 25.

De acordo com o desenvolvimento realizado para o tubo de quatro camadas, é possível realizar a mesma metodologia para tubos com $2^{*} n$ camadas. Para isso, deve-se associar as camadas duas a duas e utilizar a compatibilidade de deslocamentos e a pressão de contato de modo a acoplar essas camadas e reduzir o número de equações.

Essas matrizes de flexibilidade, desenvolvidas aqui analiticamente, podem ser utilizadas na implementação de qualquer método numérico baseado na idéia de discretização do meio contínuo, como o Método dos Elementos Finitos [14] e o Método dos Elementos de Contorno [15]. No lugar das propriedades constitutivas usuais em problemas estruturais isotrópicos, dadas pelos módulos de rigidez longitudinal e transversal e pelo Coeficiente de Poisson (que podem se generalizar assumindo valores distintos para cada direção coordenada no caso de problemas anisotrópicos) 
entram as propriedades direcionais correspondentes ao comportamento de tubos multicamadas.

\section{Conclusões}

Esta pesquisa é a etapa inicial de desenvolvimento de uma metodologia meso escala para futura análise de problemas anisotrotrópicos e heterogêneos, empregando métodos numéricos. Assim, foi necessário gerar uma formulação analítica preliminar envolvendo esforços de pressão interna em tubulações isotrópicas setorialmente homogêneas não apenas para a compreensão da física envolvida nesse tipo de problema, mas para a obtenção de um modelo matricial local que possa se compatibilizar e ser utilizado em algum dos principais métodos numéricos.

Chegou-se aqui a um equacionamento analítico conciso para a análise de uma tubulação composta por tubos de paredes espessas de diferentes materiais submetidos tanto a pressão interna quanto externa. $\mathrm{O}$ modelo é válido para sistemas compostos por $2 n$ tubos.

Em etapa posterior busca-se a expansão para sistemas compostos por $2 n+1$ tubos e também o acoplamento do efeito correspondente à solicitação de torção, mantendo-se a simetria circunferencial do modelo.

\section{Referências}

[1] EDMANS, B. D., GUO, T., ZHANG, Z., PHAM, D. C., SRIDHAR, N., \& STEWART, G.: Multiscale Modelling Approaches for Flexible Risers: Procedures, Capabilities and Demonstrations. Offshore Technology Conference. doi:10.4043/26490-MS. 22 de Março, 2016.

[2] CHATZIGEORGIOU, G.; CHARALAMBAKIS, N.; MURAT, F.: Homogenization problems of a hollowcylindermadeofelasticmaterialswithdiscontinuous properties. Int. J. Solids Struct. 45, 5165-5180 (2008)

[3] BRAGA, A.: Cilindros de Paredes Grossas (Solução de Lamé). Departamento de Engenharia Mecânica PUC Rio.
[4] XIQIA, C. et al.: A finite element analysis for unbounded flexible risers under bending loads. Ocean Systems Engineering, 2015. 77-89 p. v. 5.

[5] SHOKROLAHI-ZADEH, B., SHODJA, H.M.: Spectral equivalent inclusion method: anisotropic cylindrical multi-inhomogeneities. J. Mech. Phys. Solids, 2008. 3565-3575 p. v. 56.

[6] HONJO, K.: Thermal stresses and effective properties calculated for fiber composites using actual cylindrically-anisotropic properties of interfacial carbon coating. Carbon, 2006. 865-872 p. v. 45.

[7] GAUTAM, M. et al.: Hybrid composite tensile armour wires in flexible risers: A multi-scale model. Composite Structures, 2017. 13-27 p. v. 162.

[8] TSUKROV, I.; DRACH, B.: Elastic deformation of composite cylinders with cylindrically orthotropic layers. Int. J. Solids Structures, 2010. 25-33 p. v. 47.

[9] Tarn, J.-Q., Wang, Y.-M.: Laminated composite tubes under extension, torsion, bending, shearing and pressuring: a state space approach. Int. J. Solids Structures, 2001. 9053-9075 p. v. 38.

[10] BREBBIA, C. A. e WALKER, S.: Boundary element techniques in engineering, Newnes-Butterworths: London, 1980.

[11] BATHE, K. J.: Finite element procedures, PrenticeHall: New Jersey, 1995. 1037p.

[12] MARQUES, E.: Combinação dos métodos dos elementos de contorno e dos elementos finitos: aplicação a problemas de potencial, Dissertação de Mestrado, COPPE-UFRJ, Rio de Janeiro, 1986.

[13] FERREIRA, W.G.: Implementação de elemento de colocação não nodal para análise tridimensional pelo método dos elementos de contorno. Dissertação de Mestrado, COPPE-UFRJ, Rio de Janeiro, 1990.

[14] REDDY, J.: An Introduction to the Finite Element Method, 3rd edition, McGraw-Hill, 2005. 912p.

[15] WROBEL, L.C., ALIABADI, M.H.: "The Boundary Element Method”, Wiley, Chichester, 2002. 